\title{
Pragmatical adaptive synchronization of different orders chaotic systems with all uncertain parameters via nonlinear control
}

\author{
Shih-Yu Li • Zheng-Ming Ge
}

Received: 28 May 2010 / Accepted: 15 September 2010 / Published online: 21 October 2010

(C) The Author(s) 2010. This article is published with open access at Springerlink.com

\begin{abstract}
A new adaptive synchronization scheme by pragmatical asymptotically stability theorem is proposed in this paper. Based on this theorem and nonlinear control theory, a new adaptive synchronization scheme to design controllers can be obtained and especially the constraints for minimum values of feedback gain $K$ in controllers can be derived. This new strategy shows that the constraint values of feedback gain $K$ are related to the error of unknown and estimated parameters if the goal system is given. Through this new strategy, an appropriate feedback gain $K$ can be always decided easily to obtain controllers achieving adaptive synchronization. Two identical Lorenz systems with different initial conditions and two completely different nonlinear systems with different orders, augmented Rössler's system and Mathieu-van der Pol system, are used for illustrations to demonstrate the efficiency and effectiveness of the new adaptive scheme in numerical simulation results.
\end{abstract}

Keywords Constraints of feedback gain - Adaptive synchronization - Uncertain parameters · Nonlinear control · Pragmatical asymptotically stability theorem

S.-Y. Li · Z.-M. Ge (ه)

Department of Mechanical Engineering, National Chiao Tung University, 1001 Ta Hsueh Road, Hsinchu 300, Taiwan, Republic of China e-mail: zmg@cc.nctu.edu.tw

\section{Introduction}

Nonlinear dynamics, commonly called the chaos theory, changes the scientific way of looking at the dynamics of natural and social systems, which has been intensively studied over the past several decades. The phenomenon of chaos has attracted widespread attention amongst mathematicians, physicists and engineers. Chaos has also been extensively studied in many fields, such as chemical reactions, power converters, biological systems, information processing, secure communications, etc. [1-9].

Synchronization of chaotic systems is essential in variety of applications, including secure communication, physiology, nonlinear optics and so on. Accordingly, following the initial work of Pecora and Carroll [10] in synchronization of identical chaotic systems with different initial conditions, many approaches have been proposed for the synchronization of chaotic and hyperchaotic systems such as linear and nonlinear feedback synchronization methods [11, 12], adaptive synchronization methods [13, 14], backstepping design methods $[15,16]$, and sliding mode control methods [17, 18], etc. However, to our best knowledge, most of the methods mentioned above and many other existing synchronization methods mainly concern the synchronization of two identical chaotic or hyperchaotic systems, the methods of synchronization of two different chaotic or hyperchaotic systems are far from being straightforward because of their different structures and parameter mismatch. Moreover, 
most of the methods are used to synchronize only two systems with exactly known structures and parameters. But in practical situations, some or all of the systems' parameters cannot be exactly known in priori. As a result, more and more applications of chaos synchronization in secure communication have made it much more important to synchronize two different hyperchaotic systems with uncertain parameters in recent years. In this regard, some works on synchronization of two different hyperchaotic systems with uncertain parameters have been performed [19, 20].

In current scheme of adaptive synchronization, traditional Lyapunov stability theorem and Barbalat lemma are used to prove that the error vector approaches zero as time approaches infinity, but the question that why those estimated parameters also approach the uncertain values remains no answer [21-23]. In this article, pragmatical asymptotically stability theorem and an assumption of equal probability for ergodic initial conditions [24, 25] are used to prove strictly that those estimated parameters approach the uncertain values. Moreover, traditional adaptive chaos synchronization in general is limited for the same system.

Recently, Meng and Wang [26] proposed a new control law which is designed to achieve the generalized synchronization of chaotic systems through Barbalat lemma [27]. In [26], they also derive a synchronization condition for controllers, and the generalized synchronization between two chaotic systems can be really attained via these conditions and the control law mentioned above. In this paper, we further expand the innovative idea proposed by [26] to discuss the adaptive synchronization with all uncertain parameters in master system by pragmatical asymptotically stability theorem. Based on this theorem and nonlinear control theory, the constraints of feedback gain $K$ in controllers for adaptive synchronization are proposed and good effectiveness is shown in simulation results.

The layout of the rest of the paper is as follows. In Sect. 2, adaptive synchronization scheme is presented. In Sect. 3, two simulation cases are illustrated to verify the new adaptive scheme. In Sect. 4 conclusions are given. Pragmatical asymptotically stability theorem is enclosed in Appendix A.

\section{Adaptive synchronization scheme}

Consider the following master chaotic system:

$\dot{x}=A x+B f(x)$

where $x=\left[x_{1}, x_{2}, \ldots, x_{n}\right]^{\mathrm{T}} \in R^{n}$ denotes a state vector, $f$ is a nonlinear continuous vector function and $A$ and $B$ are $n \times n$ coefficient matrices.

The slave system is given by the following equation:

$\dot{y}=\hat{A} y+\hat{B} g(y)+u(t)$

where $y=\left[y_{1}, y_{2}, \ldots, y_{n}\right]^{\mathrm{T}} \in R^{n}$ denotes a state vector, $\hat{A}$ and $\hat{B}$ are $n \times n$ estimated coefficient matrices, $g$ is a nonlinear continuous vector function, and $u(t)=\left[u_{1}(t), u_{2}(t), \ldots, u_{n}(t)\right]^{\mathrm{T}} \in R^{n}$ is a control input vector.

Function $f(x)$ is globally Lipschitz continuous; i.e., the following condition is satisfied: For function $f(z)$, there exists constant $L>0$, for any two different $z_{1}, z_{2} \in R^{n}$, such that

$\left\|f\left(z_{1}\right)-f\left(z_{2}\right)\right\| \leq L\left\|z_{1}-z_{2}\right\|$

Property 1 [28] For a matrix $A$, let $\|A\|$ indicate the norm of $A$ induced by the Euclidean vector norm as follow:

$\|A\|=\left(\lambda_{\max }\left(A^{\mathrm{T}} A\right)\right)^{\frac{1}{2}}$

where $\lambda_{\max }\left(A^{\mathrm{T}} A\right)$ represents the maximum eigenvalue of matrix $\left(A^{\mathrm{T}} A\right)$.

Property 2 [11] For a vector,

$\|x\|=\left(x^{\mathrm{T}} x\right)^{\frac{1}{2}}$

where $x^{\mathrm{T}}$ denotes the transpose of the vector $x$.

Our goal is to design a controller $u(t)$ so that the state vector of the chaotic system (2.2) asymptotically approaches the state vector of the master system (2.1).

Theorem 1 If the parametric update laws are chosen as:

$\dot{\hat{a}}_{m}=-\tilde{a}_{m} e, \quad m=1 \sim p$

where $e=x-y$ are the state errors between the master and slave systems, $p$ is the number of parameters, 
$a_{m}$ are the unknown parameters in the master system, $\hat{a}_{m}$ are the estimated parameters in the slave system, and $\tilde{a}_{m}=a_{m}-\hat{a}_{m}$ are the errors between the unknown and estimated parameters; and the controller $u$ is designed as:

$$
\begin{aligned}
u= & -\dot{y}_{\text {out }}+K(x-y)+B f(y) \\
& +A y-\tilde{a}_{m}^{2}(e-1), \quad m=1 \sim p
\end{aligned}
$$

where $\dot{y}_{\text {out }}$ is $\dot{y}$ without controllers, gain $K=\operatorname{diag}\left(k_{1}\right.$, $\left.\ldots, k_{n}\right)$ satisfies following constraint:

$\frac{\min (K)}{\left(L\|B\|+\|A\|+\max \left(\sum_{i=1}^{p} \tilde{a}_{i}^{2}\right)\right)}>1$

then master system (2.1) and slave system (2.2) will achieve the adaptive synchronization.

Proof The synchronization errors between master and slave systems are defined

$e(t)=x(t)-y(t)$

controllers in (2.7) are substituted into the error dynamics system as follows:

$$
\begin{aligned}
\dot{e}= & \dot{x}-\dot{y} \\
= & A e+B(f(x)-f(y)) \\
& -K e+\tilde{a}_{m}^{2}(e-1), \quad m=1 \sim p
\end{aligned}
$$

We choose a control Lyapunov function as the following form:

$$
\begin{aligned}
V(t) & =\frac{1}{2}\left(e^{\mathrm{T}} e+\tilde{a}_{1}^{\mathrm{T}} \tilde{a}_{1}+\tilde{a}_{2}^{\mathrm{T}} \tilde{a}_{2}+\cdots+\tilde{a}_{p}^{\mathrm{T}} \tilde{a}_{p}\right) \\
& =\frac{1}{2}\left(\|e\|^{2}+\tilde{a}_{1}^{2}+\tilde{a}_{2}^{2}+\cdots+\tilde{a}_{p}^{2}\right)>0
\end{aligned}
$$

The time derivative of $V(t)$ in along any trajectory of (2.10) is

$$
\begin{aligned}
\dot{V}(t)= & e^{\mathrm{T}} A e+e^{\mathrm{T}} B(f(x)-f(y))-e^{\mathrm{T}} K e \\
& +e^{\mathrm{T}} \tilde{a}_{m}^{2}(e-1)+\tilde{a}_{1}^{2} e+\tilde{a}_{2}^{2} e+\cdots+\tilde{a}_{p}^{2} e \\
\leq & \|A\|\|e\|^{2}+\|e\|\|B\|\|(f(x)-f(y))\| \\
& -\min (K)\|e\|^{2}+\left(\tilde{a}_{1}^{2}+\tilde{a}_{2}^{2}+\cdots+\tilde{a}_{p}^{2}\right)\|e\|^{2} \\
\leq & \|A\|\|e\|^{2}+L\|B\|\|e\|^{2}-\min (K)\|e\|^{2} \\
& +\max \left(\tilde{a}_{1}^{2}+\tilde{a}_{2}^{2}+\cdots+\tilde{a}_{p}^{2}\right)\|e\|^{2}
\end{aligned}
$$

$$
\begin{aligned}
= & (\|A\|+L\|B\|-\min (K) \\
& \left.+\left(\tilde{a}_{10}^{2}+\tilde{a}_{20}^{2}+\cdots+\tilde{a}_{p 0}^{2}\right)\right)\|e\|^{2}
\end{aligned}
$$

where $\tilde{a}_{10}, \tilde{a}_{20}, \ldots, \tilde{a}_{p 0}$ are the initial values of $\tilde{a}_{1}, \tilde{a}_{2}$, $\ldots, \tilde{a}_{p}$, separately. If $\min (K)$ satisfies (2.8), then $\dot{V}(t) \leq 0$. Let $R=\min (K)-L\|B\|-\|A\|-\left(\tilde{a}_{10}^{2}+\right.$ $\left.\tilde{a}_{20}^{2}+\cdots+\tilde{a}_{p 0}^{2}\right)$, then $\dot{V} \leq-R\|e\|^{2}$, where $R>0$. $\dot{V}$ is a negative semidefinite function of $e$ and parameter differences. In current scheme of adaptive control of chaotic motion [21-23], traditional Lyapunov stability theorem and Barbalat lemma are used to prove the error vector approaches zero, as time approaches infinity. But the question, why the estimated or given parameters also approach to the uncertain or goal parameters, remains no answer. By pragmatical asymptotical stability theorem, the question can be answered strictly.

\section{Numerical simulations}

In this section, there are two examples for our new adaptive scheme in numerical simulation. In Case 1, two identical Lorenz systems with different initial conditions and parameters are used for master and slave systems to show the effectiveness of the new scheme. In Case 2, two completely different systems, Rössler's system and Mathieu-van der Pol system, are regarded as master and slave systems separately.

Case 1 Two identical Lorenz systems with different initial conditions and parameters.

Master Lorenz system [29]:

$$
\begin{aligned}
{\left[\begin{array}{c}
\dot{x}_{1} \\
\dot{x}_{2} \\
\dot{x}_{3}
\end{array}\right]=} & {\left[\begin{array}{c}
a\left(x_{2}-x_{1}\right) \\
c x_{1}-x_{1} x_{3}-x_{2} \\
x_{1} x_{2}-b x_{3}
\end{array}\right] } \\
= & {\left[\begin{array}{ccc}
-a & a & 0 \\
c & -1 & 0 \\
0 & 0 & -b
\end{array}\right]\left[\begin{array}{l}
x_{1} \\
x_{2} \\
x_{3}
\end{array}\right] } \\
& +\left[\begin{array}{ccc}
0 & 0 & 0 \\
0 & -1 & 0 \\
0 & 0 & 1
\end{array}\right]\left[\begin{array}{c}
0 \\
x_{1} x_{3} \\
x_{1} x_{2}
\end{array}\right]
\end{aligned}
$$

where

$$
A=\left[\begin{array}{ccc}
-a & a & 0 \\
c & -1 & 0 \\
0 & 0 & -b
\end{array}\right]
$$


$B=\left[\begin{array}{ccc}0 & 0 & 0 \\ 0 & -1 & 0 \\ 0 & 0 & 1\end{array}\right]$ and $f(x)=\left[\begin{array}{c}0 \\ x_{1} x_{3} \\ x_{1} x_{2}\end{array}\right]$

$x_{1}, x_{2}, x_{3}$ are states of master system, when initial condition $\left(x_{10}, x_{20}, x_{30}\right)=(-0.1,0.2,0.3)$ and parameters $a=10, b=8 / 3$ and $c=28$. The chaotic behavior of (3.1) is shown in Fig. 1.

Slave Lorenz system:

$$
\left[\begin{array}{c}
\dot{y}_{1} \\
\dot{y}_{2} \\
\dot{y}_{3}
\end{array}\right]=\left[\begin{array}{c}
\hat{a}\left(y_{2}-y_{1}\right)+u_{1} \\
\hat{c} y_{1}-y_{1} y_{3}-y_{2}+u_{2} \\
y_{1} y_{2}-\hat{b} y_{3}+u_{3}
\end{array}\right]
$$

$y_{1}, y_{2}, y_{3}$ are states of slave system, when initial condition $\left(y_{10}, y_{20}, y_{30}\right)=(5,10,5)$ and initial estimated parameters $\hat{a}_{0}=8, \hat{b}_{0}=3$ and $\hat{c}_{0}=25$. Thus, the errors between the estimated and uncertain parameters can be given as follows:

$\left\{\begin{array}{l}\tilde{a}_{0}=a-\hat{a}_{0}=2 \\ \tilde{b}_{0}=b-\hat{b}_{0}=-1 / 3 \\ \tilde{c}_{0}=c-\hat{c}_{0}=3\end{array}\right.$

Through (3.1) and (3.3), $\|A\|=30.0731,\|B\|=1$, and $\max \left(\tilde{a}^{2}+\tilde{b}^{2}+\tilde{c}^{2}\right) \cong 13.111$ can be obtained. According to our new adaptive scheme, gain $K$ should satisfy (2.8), i.e.,

$\frac{\min (K)}{(L \times 1+30.0731+13.111)}>1$

choosing $L=1$, the gain matrix can be selected as follows:

$K=\left[\begin{array}{ccc}55 & 0 & 0 \\ 0 & 52 & 0 \\ 0 & 0 & 50\end{array}\right]$

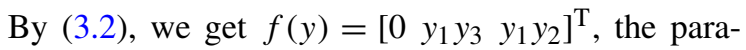
metric update laws and corresponding controllers can be decided by (2.6) and (2.7). Then we can obtain the time derivative of $V(t)$ is semidefinite as follow:

$$
\begin{aligned}
\dot{V} \leq & (\|A\|+L\|B\|-\min (K) \\
& \left.+\max \left(\tilde{a}_{0}^{2}+\tilde{b}_{0}^{2}+\tilde{c}_{0}^{2}\right)\right)\|e\|^{2} \\
= & (44.1842-50)\|e\|^{2} \\
= & (-5.8158)\|e\|^{2}<0
\end{aligned}
$$

which is a negative semidefinite function of $e_{1}, e_{2}, e_{3}$, $\tilde{a}, \tilde{b}, \tilde{c}$. The Lyapunov asymptotical stability theorem is not satisfied. We cannot obtain that common origin of error dynamics and parameter dynamics is asymptotically stable. By pragmatical asymptotically stabil-
Fig. 1 Projections of phase portrait of chaotic Lorenz system with $a=10$, $b=8 / 3$, and $c=28$
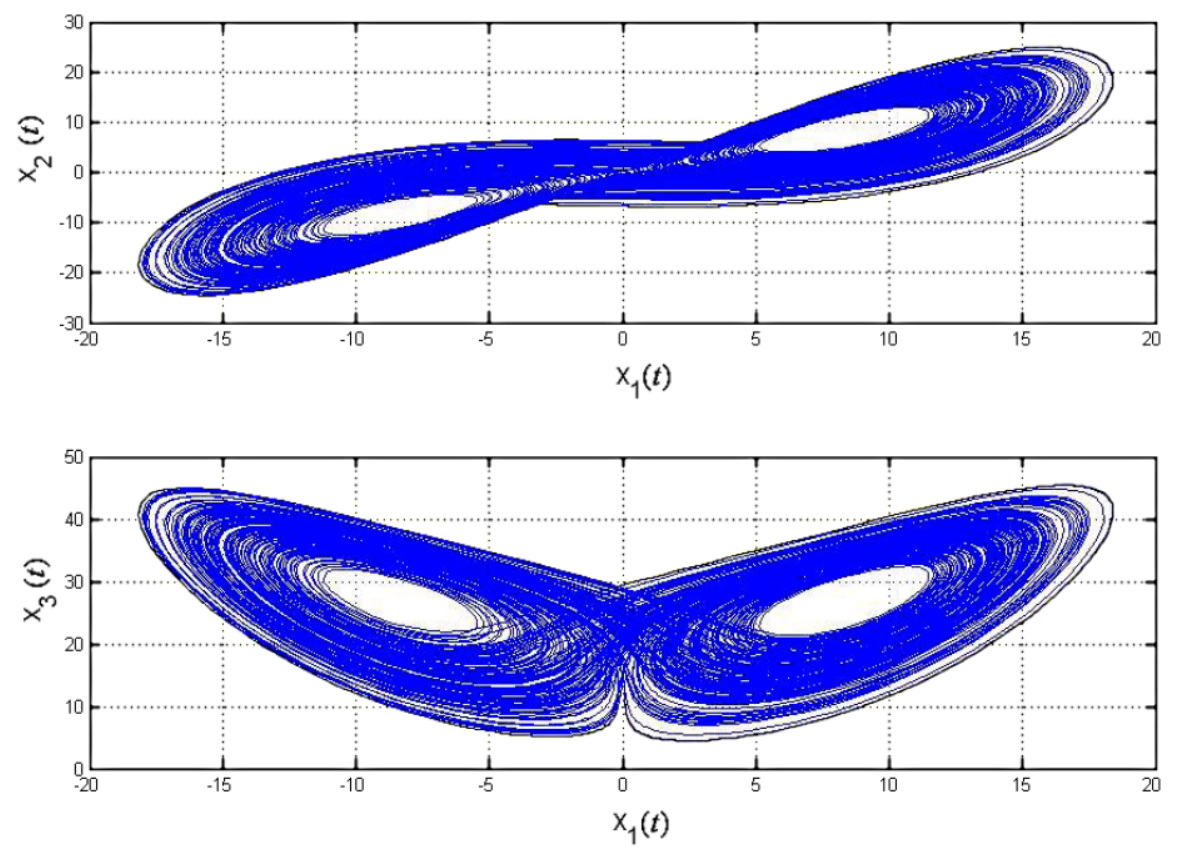
Fig. 2 Time histories of errors for Case 1

Fig. 3 Time histories of parametric errors for Case 1
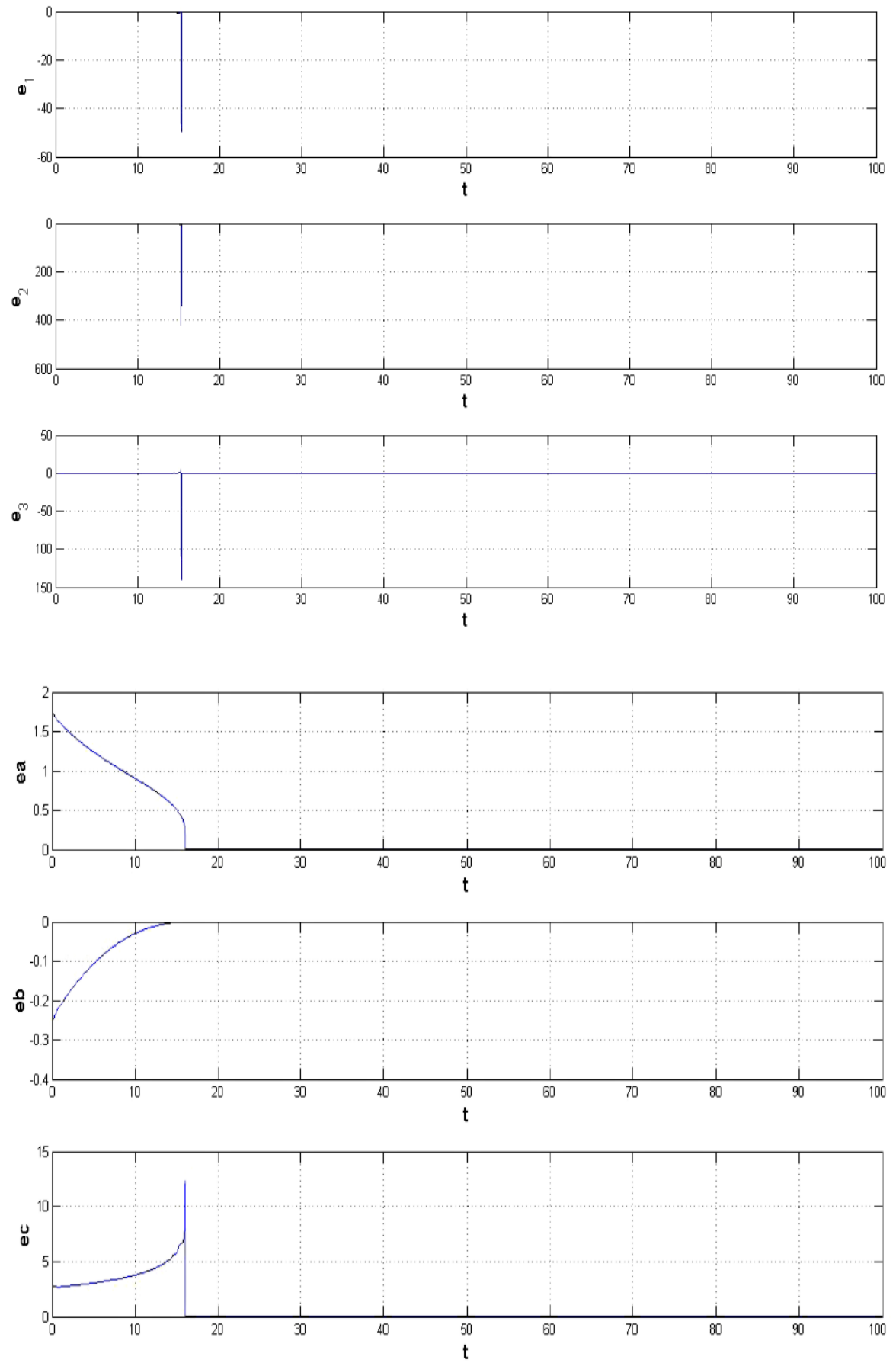

ity theorem (see Appendix A), $D$ is a 6-manifold, $n=6$, and the number of error state variables $p=3$. When $e_{1}=e_{2}=e_{3}=0$ and $\hat{a}, \hat{b}, \hat{c}$ take arbitrary values, $\dot{V}=0$, so $X$ is of 3 dimensions, $m=n-p=6-$ $3=3, m+1<n$ is satisfied. According to the pragmatical asymptotically stability theorem, error vector $e$ approaches zero and the estimated parameters also approach the uncertain parameters. The equilibrium point is pragmatically asymptotically stable. Under the assumption of equal probability, it is actually asymptotically stable. The simulation results are shown in Figs. 2 and 3. 
Fig. 4 Projections of phase portrait of chaotic Rössler's system with $a=0.2$, $b=0.2$, and $c=5.7$
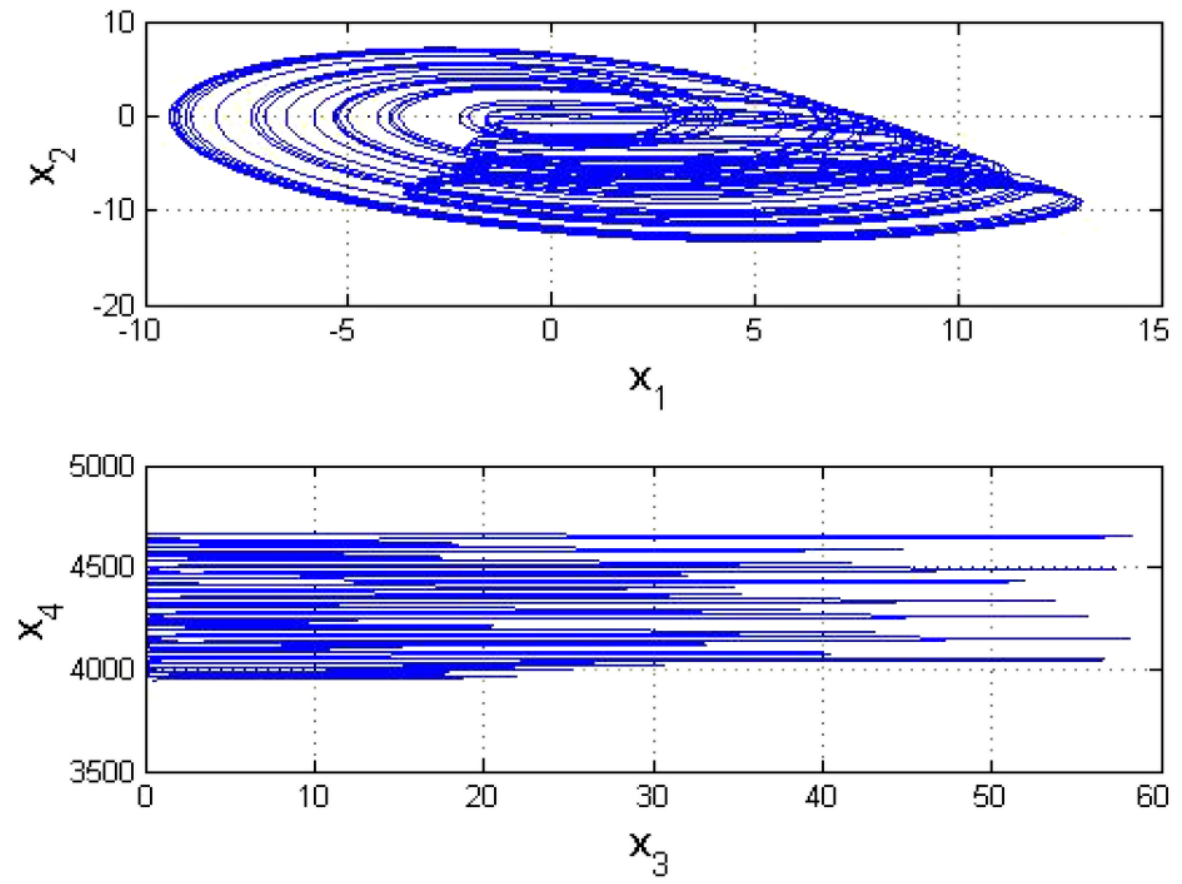

Case 2 Adaptive synchronization between augmented new Rössler's system as a master system and Mathieu-van der Pol system as a slave one.

The augmented new Rössler's system with four orders is:

$$
\begin{aligned}
{\left[\begin{array}{c}
\dot{x}_{1} \\
\dot{x}_{2} \\
\dot{x}_{3} \\
\dot{x}_{4}
\end{array}\right]=} & {\left[\begin{array}{c}
-x_{2}-x_{3} \\
x_{1}+a x_{2} \\
b+x_{1} x_{3}-c x_{3} \\
x_{1}+x_{3}
\end{array}\right] } \\
= & {\left[\begin{array}{cccc}
0 & -1 & -1 & 0 \\
1 & a & 0 & 0 \\
0 & 0 & -c & 0 \\
1 & 0 & 1 & 0
\end{array}\right]\left[\begin{array}{c}
x_{1} \\
x_{2} \\
x_{3} \\
x_{4}
\end{array}\right] } \\
& +\left[\begin{array}{llll}
0 & 0 & 0 & 0 \\
0 & 0 & 0 & 0 \\
0 & 0 & 1 & 0 \\
0 & 0 & 0 & 0
\end{array}\right]\left[\begin{array}{c}
0 \\
0 \\
b+x_{1} x_{3} \\
0
\end{array}\right]
\end{aligned}
$$

where

$$
A=\left[\begin{array}{cccc}
0 & -1 & -1 & 0 \\
1 & a & 0 & 0 \\
0 & 0 & -c & 0 \\
1 & 0 & 1 & 0
\end{array}\right]
$$

$B=\left[\begin{array}{llll}0 & 0 & 0 & 0 \\ 0 & 0 & 0 & 0 \\ 0 & 0 & 1 & 0 \\ 0 & 0 & 0 & 0\end{array}\right]$ and $f(x)=\left[\begin{array}{c}0 \\ 0 \\ b+x_{1} x_{3} \\ 0\end{array}\right]$

$x_{1}, x_{2}, x_{3}$ and $x_{4}$ are states of new Rössler's system. We choose initial condition $\left(x_{10}, x_{20}, x_{30}, x_{40}\right)=$ $(0.1,0.5,0.3,0.7)$ and parameters $a=0.38, b=0.3$, and $c=4.820$. Chaos of the new Rössler's system appears. The chaotic behavior of (3.7) is shown in Fig. 4.

Slave Mathieu-van der Pol system [30] is

$$
\left[\begin{array}{c}
\dot{z}_{1} \\
\dot{z}_{2} \\
\dot{z}_{3} \\
\dot{z}_{4}
\end{array}\right]=\left[\begin{array}{c}
z_{2}+u_{1} \\
-\left(\hat{a}_{1}+\hat{b}_{1} z_{3}\right) z_{1}-\left(\hat{a}_{1}+\hat{b}_{1} z_{3}\right) z_{1}^{3}-\hat{c}_{1} z_{2}+\hat{d}_{1} z_{3}+u_{2} \\
z_{4}+u_{3} \\
-\hat{e}_{1} z_{3}+\hat{f}_{1}\left(1-z_{3}^{2}\right) z_{4}+\hat{g}_{1} z_{1}+u_{4}
\end{array}\right]
$$

$z_{1}, z_{2}, z_{3}$ and $z_{4}$ are states of slave system, $\hat{a}_{1}, \hat{b}_{1}, \hat{c}_{1}$, $\hat{d}_{1}, \hat{e}_{1}, \hat{f}_{1}$ and $\hat{g}_{1}$ are uncertain parameters. This system exhibits chaos when the parameters of system are $\hat{a}_{1}=10, \hat{b}_{1}=3, \hat{c}_{1}=0.4, \hat{d}_{1}=70, \hat{e}_{1}=1$, $\hat{f}_{1}=5, \hat{g}_{1}=0.1$ and the initial states of system are $\left(z_{10}, z_{20}, z_{30}, z_{40}\right)=(0.1,-0.5,0.1,-0.5)$. The projections of phase portraits are shown in Fig. 5. We choose $\hat{a}_{1}=3, \hat{b}_{1}=1, \hat{c}_{1}=0.4, \hat{d}_{1}=5, \hat{e}_{1}=1, \hat{f}_{1}=$ $1, \hat{g}_{1}=0.1, \hat{a}=0, \hat{b}=0, \hat{c}=0$ and the initial states 
Fig. 5 Projections of phase portrait of new chaotic Mathieu-van der Pol system with $\hat{a}_{1}=10$, $\hat{b}_{1}=3, \hat{c}_{1}=0.4, \hat{d}_{1}=70$, $\hat{e}_{1}=1, \hat{f}_{1}=5, \hat{g}_{1}=0.1$
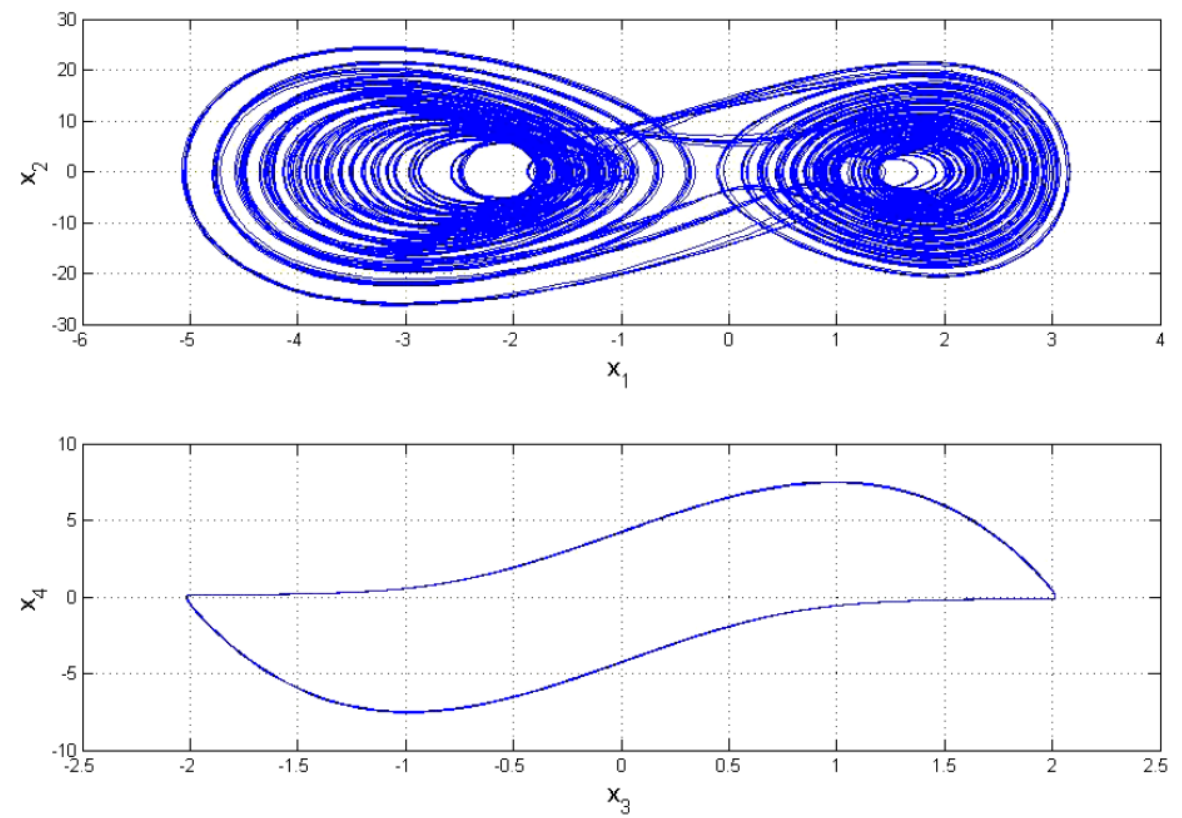

of system are $\left(z_{10}, z_{20}, z_{30}, z_{40}\right)=(5,7,9,10)$. The initial values of $\hat{a}_{10}, \hat{b}_{10}, \hat{c}_{10}, \hat{d}_{10}, \hat{e}_{10}, \hat{f}_{10}, \hat{g}_{10}, \hat{a}_{0}, \hat{b}_{0}$ and $\hat{c}_{0}$ can be decided as: $\hat{a}_{10}=3, \hat{b}_{10}=1, \hat{c}_{10}=0.4$, $\hat{d}_{10}=5, \hat{e}_{10}=0.3, \hat{f}_{10}=1, \hat{g}_{10}=0.1, \hat{a}_{0}=0, \hat{b}_{0}=0$ and $\hat{c}_{0}=0$. Therefore, the errors of the estimated unknown parameters can be decided as follow:

$$
\left\{\begin{array}{l}
\tilde{a}_{10}=a_{1}-\hat{a}_{10}=-3 \\
\tilde{b}_{10}=b_{1}-\hat{b}_{10}=-1 \\
\tilde{c}_{10}=c_{1}-\hat{c}_{10}=-0.4 \\
\tilde{d}_{10}=d_{1}-\hat{d}_{10}=-5 \\
\tilde{e}_{10}=e_{1}-\hat{e}_{10}=-1 \\
\tilde{f}_{10}=f_{1}-\hat{f}_{10}=-1 \\
\tilde{g}_{10}=g_{1}-\hat{g}_{10}=-0.1 \\
\tilde{a}_{0}=a-\hat{a}_{0}=0.2 \\
\tilde{b}_{0}=b-\hat{b}_{0}=0.2 \\
\tilde{c}_{0}=c-\hat{c}_{0}=5.7
\end{array}\right.
$$

Through (3.7) and (3.9), $\|A\|=5.8780,\|B\|=1$, and $\max \left(\tilde{a}^{2}+\tilde{b}^{2}+\tilde{c}^{2}+\tilde{a}_{1}^{2}+\cdots+\tilde{g}_{1}^{2}\right)=68.9300$ can be obtained. According to our new adaptive scheme, gain $K$ must satisfy (2.8), i.e.

$$
\frac{\min (K)}{(L \times 1+5.8780+68.9300)}>1
$$

Choose $L=1$. The gain matrix can be selected as follow:

$$
K=\left[\begin{array}{cccc}
90 & 0 & 0 & 0 \\
0 & 87 & 0 & 0 \\
0 & 0 & 83 & 0 \\
0 & 0 & 0 & 80
\end{array}\right]
$$

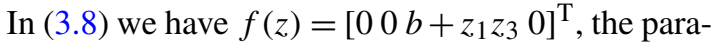
metric update laws and corresponding controllers can be decided by (2.6) and (2.7). Then we can obtain the time derivative of $V(t)$ is negative semidefinite as follow:

$$
\begin{aligned}
\dot{V} \leq & (\|A\|+L\|B\|-\min (K) \\
& \left.+\max \left(\tilde{a}^{2}+\tilde{b}^{2}+\tilde{c}^{2}+\tilde{a}_{1}^{2}+\cdots+\tilde{g}_{1}^{2}\right)\right)\|e\|^{2} \\
= & (75.7080-80)\|e\|^{2} \\
= & (-4.2920)\|e\|^{2}<0
\end{aligned}
$$

which is a negative semidefinite function of $e_{1}, e_{2}, e_{3}$, $e_{4}, \tilde{a}, \tilde{b}, \tilde{c}, \tilde{a}_{1}, \tilde{b}_{1}, \tilde{c}_{1}, \tilde{d}_{1}, \tilde{e}_{1}, \tilde{f}_{1}, \tilde{g}_{1}$. The Lyapunov asymptotical stability theorem is not satisfied. We cannot obtain that common origin of error dynamics and parameter dynamics is asymptotically stable. By pragmatical asymptotically stability theorem (see Appendix A), D is a 14-manifold, $n=14$ and the number of error state variables $p=4$. When $e_{1}=e_{2}=$ $e_{3}=e_{4}=0$ and $\hat{a}, \hat{b}, \hat{c}, \hat{a}_{1}, \hat{b}_{1}, \hat{c}_{1}, \hat{d}_{1}, \hat{e}_{1}, \hat{f}_{1}$ and $\hat{g}_{1}$ 
Fig. 6 Time histories of errors for Case 2

Fig. 7 Time histories of parametric errors $\tilde{a}, \tilde{c}, \tilde{a}_{1}$ and $\tilde{b}_{1}$ for Case 2
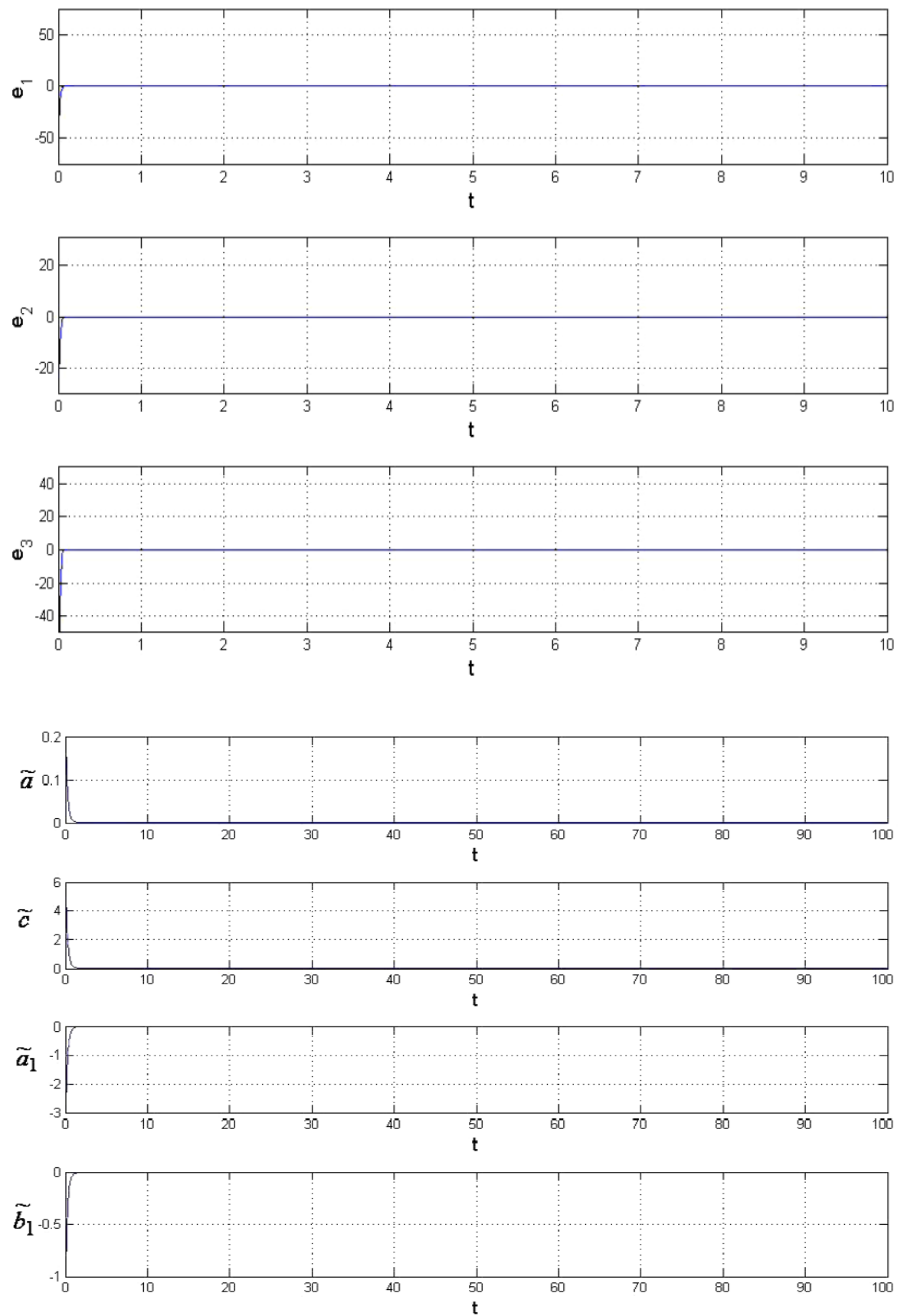

take arbitrary values, $\dot{V}=0$, so $X$ is of 4 dimensions, $m=n-p=14-4=10, m+1<n$ is satisfied. According to the pragmatical asymptotically stability theorem, error vector e approaches zero and the estimated parameters also approach the uncertain parameters. The equilibrium point is pragmatically asymptotically stable. Under the assumption of equal probability, it is actually asymptotically sta- ble. The simulation results are shown in Figs. 6, 7 and 8.

\section{Conclusions}

In this paper, a new adaptive synchronization scheme which is derived by pragmatical asymptotically stabil- 
Fig. 8 Time histories of parametric errors $\tilde{c}_{1}, \tilde{d}_{1}, \tilde{e}_{1}, \tilde{f}_{1}$ and $\tilde{g}_{1}$ for Case 2
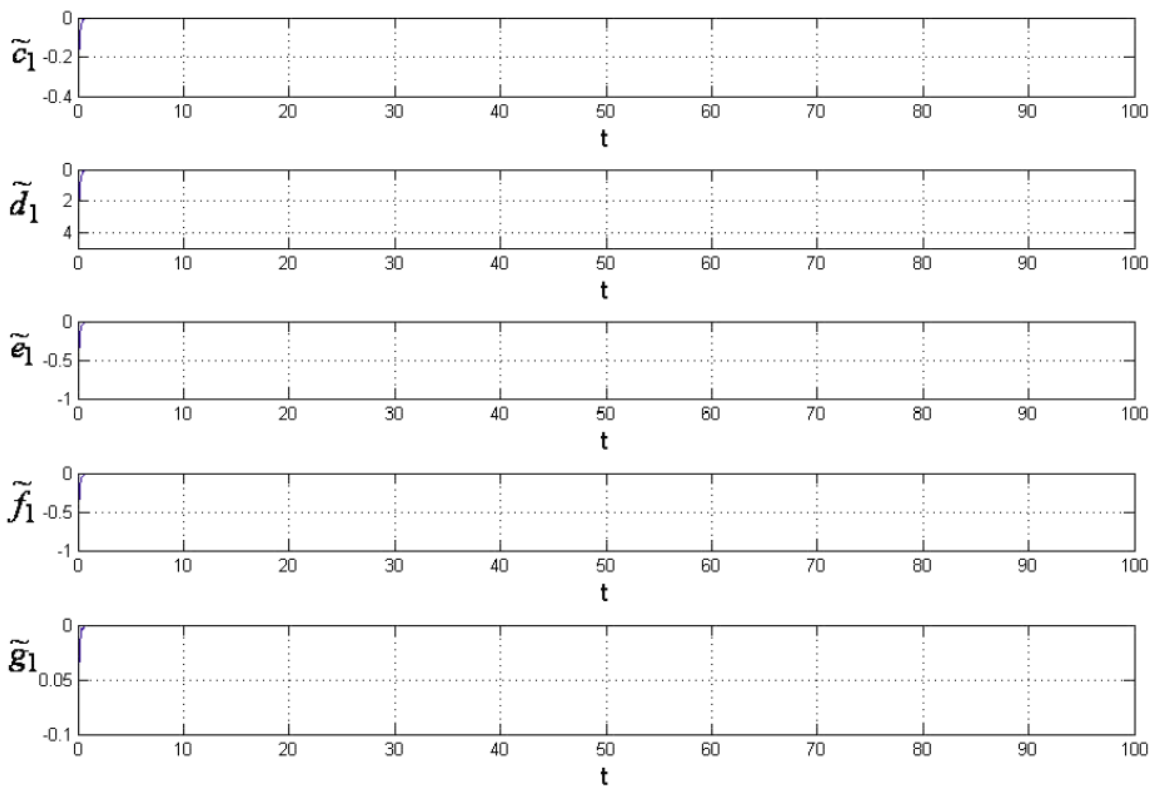

ity theorem is proposed to achieve adaptive synchronization. We give a simple and useful result in this article as: If the master system is given, the minimum values in feedback gain $K$ can be calculated by the sum of errors between unknown and estimated parameters, i.e. our new strategy proves the existence of relation between the constraint values of feedback gain $K$ and the sum of the errors of unknown parameters and estimated parameters. By applying this new relation formula, an appropriate feedback gain $K$ can be decided easily to obtain controllers achieving adaptive synchronization. Simulation results show that not only for two identical nonlinear systems with all unknown parameters adaptive synchronization can be achieved, but also for two completely different nonlinear systems with different orders and all unknown parameters this goal can be attained. Therefore, the new adaptive scheme is really useful and effective in adaptive synchronization of various kinds of different nonlinear systems.

Acknowledgements This research was supported by the National Science Council, Republic of China, under Grant Number NSC 96-2221-E-009-145-MY3.

Open Access This article is distributed under the terms of the Creative Commons Attribution Noncommercial License which permits any noncommercial use, distribution, and reproduction in any medium, provided the original author(s) and source are credited.

\section{Appendix A: Pragmatical asymptotical stability theory}

The stability for many problems in real dynamical systems is actual asymptotical stability, although may not be mathematical asymptotical stability. The mathematical asymptotical stability demands that trajectories from all initial states in the neighborhood of zero solution must approach the origin as $t \rightarrow \infty$. If there are only a small part or even a few of the initial states from which the trajectories do not approach the origin as $t \rightarrow \infty$, the zero solution is not mathematically asymptotically stable. However, when the probability of occurrence of an event is zero, it means the event does not occur actually. If the probability of occurrence of the event that the trajectories from the initial states are that they do not approach zero when $t \rightarrow \infty$, is zero, the stability of zero solution is actual asymptotical stability though it is not mathematical asymptotical stability. In order to analyze the asymptotical stability of the equilibrium point of such systems, the pragmatical asymptotical stability theorem is used.

Let $X$ and $Y$ be two manifolds of dimensions $m$ and $n(m<n)$, respectively, and $\varphi$ be a differentiable map from $X$ to $Y$, then $\varphi(X)$ is subset of Lebesque measure 0 of $Y$ [31]. For an autonomous system,

$\frac{d x}{d t}=f\left(x_{1}, \ldots, x_{n}\right)$ 
where $x=\left[x_{1}, \ldots, x_{n}\right]^{\mathrm{T}}$ is a state vector, the function $f=\left[f_{1}, \ldots, f_{n}\right]^{\mathrm{T}}$ is defined on $D \subset R^{n}$ and $\|x\| \leq H>0$. Let $x=0$ be an equilibrium point for the system (A.1). Then

$f(0)=0$

For a nonautonomous systems,

$\dot{x}=f\left(x_{1}, \ldots, x_{n+1}\right)$

where $x=\left[x_{1}, \ldots, x_{n+1}\right]^{\mathrm{T}}$, the function $f=$ $\left.f_{1}, \ldots, f_{n}\right]^{\mathrm{T}}$ is defined on $D \subset R^{n} \times R_{+}$here $t=$ $x_{n+1} \subset R_{+}$. The equilibrium point is

$f\left(0, x_{n+1}\right)=0$

Definition The equilibrium point for the system (A.1) is pragmatically asymptotically stable provided that with initial points on $C$ which is a subset of Lebesque measure 0 of $D$, the behaviors of the corresponding trajectories cannot be determined, while with initial points on $D-C$, the corresponding trajectories behave as that agree with traditional asymptotical stability [21-23].

Theorem Let $V=\left[x_{1}, \ldots, x_{n}\right]^{\mathrm{T}}: D \rightarrow R_{+}$be positive definite and analytic on $D$, where $x_{1}, x_{2}, \ldots, x_{n}$ are all space coordinates such that the derivative of $V$ through (A.1) or (A.3), $\dot{V}$, is negative semidefinite of $\left[x_{1}, x_{2}, \ldots, x_{n}\right]^{\mathrm{T}}$.

For autonomous system, let $X$ be the m-manifold consisted of point set for which $\forall x \neq 0, \dot{V}(x)=0$ and $D$ is a $n$-manifold. If $m+1<n$, then the equilibrium point of the system is pragmatically asymptotically stable.

For nonautonomous system, let $X$ be the $m+1$ manifold consisting of point set of which $\forall x \neq 0$, $\dot{V}\left(x_{1}, x_{2}, \ldots, x_{n}\right)=0$ and $D$ is $n+1$-manifold. If $m+1+1<n+1$, i.e., $m+1<n$ then the equilibrium point of the system is pragmatically asymptotically stable. Therefore, for both autonomous and nonautonomous system, the formula $m+1<n$ is universal. So the following proof is only for autonomous system. The proof for nonautonomous system is similar.

Proof Since every point of $X$ can be passed by a trajectory of (A.1), which is onedimensional, the collection of these trajectories, $A$, is a $(m+1)$-manifold $[24,25]$.
If $m+1<n$, then the collection $C$ is a subset of Lebesque measure 0 of $D$. By the above definition, the equilibrium point of the system is pragmatically asymptotically stable.

If an initial point is ergodically chosen in $D$, the probability of that the initial point falls on the collection $C$ is zero. Here, equal probability is assumed for every point chosen as an initial point in the neighborhood of the equilibrium point. Hence, the event that the initial point is chosen from collection $C$ does not occur actually. Therefore, under the equal probability assumption, pragmatical asymptotical stability becomes actual asymptotical stability. When the initial point falls on $D-C, \dot{V}(x)<0$, the corresponding trajectories behave as they agree with traditional asymptotical stability because by the existence and uniqueness of the solution of initial-value problem, these trajectories never meet $C$.

In (2.11), $V$ is a positive definite function of $n$ variables, i.e., $p$ error state variables and $n-p=m$ differences between unknown and estimated parameters, while $\dot{V}=e^{\mathrm{T}} C e$ is a negative semidefinite function of $n$ variables. Since the number of error state variables is always more than one, $p>1, m+1<n$ is always satisfied, by pragmatical asymptotical stability theorem, we have

$\lim _{t \rightarrow \infty} e=0$

and the estimated parameters approach the uncertain parameters. The pragmatical adaptive control theorem is obtained. Therefore, the equilibrium point of the system is pragmatically asymptotically stable. Under the equal probability assumption, it is actually asymptotically stable for both error state variables and parameter variables.

\section{References}

1. Cang, S., Qi, G., Chen, Z.: Four-wing hyper-chaotic attractor and transient chaos generated from a new 4-D quadratic autonomous system. Nonlinear Dyn. 59, 515-527 (2010)

2. Lu, W.G., Zhou, L.W., Luo, Q.M., Zhang, X.F.: Filter based non-invasive control of chaos in Buck converter. Phys. Lett. A 372, 3217-3222 (2008)

3. Iannelli, L., Johansson, K.H., Jönsson, U.T., Vasca, F.: Subtleties in the averaging of a class of hybrid systems with applications to power converters. Control Eng. Pract. 16, 961-975 (2008)

4. Kazantzis, N., Kravaris, C., Syrou, L.: A new model reduction method for nonlinear dynamical systems. Nonlinear Dyn. 59, 183-194 (2010) 
5. May, R.M.: Chaos and the dynamics of biological populations. Nucl. Phys. B, Proc. Suppl. 2, 225-245 (1987)

6. Ma, J., Li, A.B., Pu, Z.S., Yang, L.J., Wang, Y.Z.: A timevarying hyperchaotic system and its realization in circuit. Nonlinear Dyn. (2010) doi:10.1007/s11071-010-9739-x

7. Song, A., Duan, J., Wu, J., Li, H.: Design 2D nonlinear system for information storage. Chaos Solitons Fractals 41, 157-163 (2009)

8. Yu, W.: High-gain observer for chaotic synchronization and secure communication. Int. J. Commun. Syst. 18, 487-500 (2005)

9. Wang, X., Zhang, J.: Chaotic secure communication based on nonlinear autoregressive filter with changeable parameters. Phys. Lett. A 357, 323-329 (2006)

10. Pecora, L.M., Carroll, T.L.: Synchronization in chaotic systems. Phys. Rev. Lett. 64(8), 821-824 (1990)

11. Zhou, B., Duan, G.R.: A novel nested non-linear feedback law for global stabilisation of linear systems with bounded controls. Int. J. Control 81(9), 1352-1363 (2008)

12. Wu, X., Chen, G., Cai, J.: Chaos synchronization of the master-slave generalized Lorenz systems via linear state error feedback control. Physica D 229, 52-80 (2007)

13. Wang, Z.L., Shi, X.R.: Adaptive Q-S synchronization of non-identical chaotic systems with unknown parameters. Nonlinear Dyn. 59, 559-567 (2010)

14. Lee, T.C., Chen, B.S., Chang, Y.C.: Adaptive control of robots by linear time-varying dynamic position feedback. Int J. Adapt. Control Signal Process. 10, 649-671 (1996)

15. Ozbay, U., Zergeroglu, E., Sivrioglu, S.: Adaptive backstepping control of variable speed wind turbines. Int. J. Control 81(6), 910-919 (2008)

16. Bowong, S.: Adaptive synchronization of chaotic systems with unknown bounded uncertainties via backstepping approach. Nonlinear Dyn. 49, 59-70 (2007)

17. Yagiz, N., Hacioglu, Y.: Robust control of a spatial robot using fuzzy sliding modes. Math. Comput. Model. 49, 114127 (2009)

18. Efe, M.Ö., Kasnakolu, C.: A fractional adaptation law for sliding mode control. Int. J. Adapt. Control Signal Process. 22, 968-986 (2008)
19. Li, Y., Liu, X., Zhang, H.: Dynamical analysis and impulsive control of a new hyperchaotic system. Math. Comput. Model. 42, 1359-1374 (2005)

20. Huang, J.: Adaptive synchronization between different hyperchaotic systems with fully uncertain parameters. Phys. Lett. A 372, 4799-4804 (2008)

21. Lu, J., Cao, J.: Adaptive synchronization in tree-like dynamical networks. Nonlinear Anal. Theory Methods Appl. 8, 1252-1260 (2007)

22. Liu, Y.J., Zheng, Y.Q.: Adaptive robust fuzzy control for a class of uncertain chaotic systems. Nonlinear Dyn. 75, 431-439 (2007)

23. Odibat, Z.M.: Adaptive feedback control and synchronization of non-identical chaotic fractional order systems. Nonlinear Dyn. 60, 479-487 (2010)

24. Ge, Z.M., Yu, J.K., Chen, Y.T.: Pragmatical asymptotical stability theorem with application to satellite system. Jpn. J. Appl. Phys. 38, 6178-6179 (1999)

25. Ge, Z.M., Yu, J.K.: Pragmatical asymptotical stability theorem on partial region and for partial variable with applications to gyroscopic systems. Chin. J. Mech. 16(4) 179-187 (2000)

26. Meng, J., Wang, X.: Generalized synchronization via nonlinear control. Chaos 18, 1054-1500 (2008)

27. Gopalsamy, K.: Stability and Oscillations in Delay Differential Equations of Population Dynamics. Kluwer Academic, Dordrecht (1992)

28. Khalil, H.K.: Nonlinear Systems, 3rd edn. Prentice Hall, New Jersey (2002)

29. Dowell, E.H.: Chaotic oscillations in mechanical systems. Comput. Mech. 3, 199-216 (1988)

30. Ge, Z.M., Li, S.Y.: Chaos control of new Mathieu-van der Pol systems with new Mathieu-Duffing systems as functional system by GYC partial region stability theory. Nonlinear Anal. Theory Methods Appl. 71, 4047-4059 (2009)

31. Matsushima, Y.: Differentiable Manifolds. Marcel Dekker, New York (1972) 